\title{
Picturing Recreation: Newcomers' Perspectives on Experiences of Recreation
}

\author{
Heidi Lauckner ${ }^{1}$ (D) Karen Gallant ${ }^{2}$ (D) Maryam Akbari $^{1} \cdot$ Giana Tomas $^{2}$ (D) \\ Tara Pride (nee White) ${ }^{1} \mathbb{D} \cdot$ Susan Hutchinson ${ }^{2} \mathbb{D}$
}

Accepted: 20 November 2021 / Published online: 29 January 2022

(c) The Author(s), under exclusive licence to Springer Nature B.V. 2021, corrected publication 2022

\begin{abstract}
The process of resettlement in a new country represents a significant transition in a person's or family's life, during which there are many changes to their daily activities. While involvement in recreational activities may support such transitions, further exploration of leisure experiences, as defined subjectively by newcomers themselves, is needed. Using an exploratory, community-based participatory approach drawing on photovoice methods, focus groups, and individual interviews, this research project explored the meanings of recreation among newcomers in two communities, one rural and one urban, in Eastern Canada. Forty newcomers $(n=40)$, originally from 13 different countries, participated in the photovoice activities. Transcripts from three focus groups and five individual interviews were analyzed, first by site to create the photo exhibits and then across sites. Across the two sites, four sub-themes were identified: (1) continuity with, and freedom from, past activities and places; (2) being in and connecting with nature; (3) staying physically and mentally well; and (4) connecting and learning with others through reciprocity. These all contributed to the overarching theme developing a sense of belonging: a series of small encounters. The findings highlight the powerful role of recreation within the resettlement process, and highlight particularly the importance of small, informal recreational experiences that are woven into everyday lives and routines. Such experiences contribute to a sense of belonging for newcomers, thus assisting the resettlement process.
\end{abstract}

Keywords Canada Immigration $\cdot$ Leisure $\cdot$ Photovoice $\cdot$ Recreation $\cdot$ Resettlement . Transitions

Heidi Lauckner

Heidi.lauckner@dal.ca

1 School of Occupational Therapy, Dalhousie University, Halifax, NS, Canada

2 School of Health and Human Performance, Dalhousie University, Halifax, NS, Canada 


\section{Introduction}

Canada is among the many countries that have welcomed increasing numbers of newcomers, guided by both the economic benefits of immigration and commitments to upholding human rights for those facing forced migration. The process of resettlement in a new country represents a significant transition in a person's or family's life. Transitions refer to processes of change; during such changes, people's daily activities, including their work, family life, and free-time activities, can be disrupted (Crider et al., 2015). In the context of resettlement, transitions can include changes to or abandonment of previous activities and engagement in new activities altogether (Schisler \& Polatajko, 2002; Suleman \& Whiteford, 2013). While housing, employment, and schooling are essential aspects of this transition (Ghahari et al., 2019), involvement in recreational activities can also facilitate settlement (Gallant \& Tirone, 2017; Perez et al., 2010), through the development of social networks and language skills (Suto, 2013). Involvement in meaningful and enjoyable activities (i.e. recreation and leisure) in community settings can be a means through which newcomers become a part of, and contribute to, the cultural life of their communities in their new contexts, while also building social networks, managing stress, and being physically active. Despite the known benefits of leisure for the general population (Caldwell, 2005), and for newcomers specifically (Gallant \& Tirone, 2017), the meanings newcomers associate with their recreation activities are not well understood, which can result in the under-utilization of subjectively meaningful recreational activities to support resettlement transitions. Further exploration of leisure experiences of newcomers can enhance our understanding of how involvement in community recreation, as defined subjectively by newcomers themselves, can contribute to resettlement. In particular, a broad definition of recreation provided a foundation for this project, to be inclusive of diverse experiences that extend beyond structured recreation (such as organized recreation programs) to explore less formal and unstructured leisure activities during resettlement processes.

Using an exploratory, community-based participatory approach (Minkler \& Wallerstein, 2011) that drew on photovoice methods, focus groups, and individual interviews, this research project explored the varied meanings of recreation among newcomers from diverse cultural backgrounds in two communities, one rural and one urban, in Eastern Canada. This project served as a foundation for a broader project aimed to support the leisure needs of newcomers by providing a means to build a common understanding of the varied meanings and experiences of recreation for newcomers. We first briefly discuss terminology, review relevant literature, and then describe our project and findings. 


\section{Terminology}

We adopted the term newcomers to describe our participants - immigrants and refugees who resettled in Canada from various countries - and specifically chose not to use the terms immigrants or refugees, given the diverse pathways they may have used to come to Canada (Caidi \& Allard, 2005; Statistics Canada, 2010). We instead use the term newcomers to collectively describe those who are recognized as being new residents to Canada and have been living in Canada for 5 years or less (Barrick et al., 2021; Caidi \& Allard, 2005) to highlight the "newness" of their experiences in Canada and to capture their broad yet unique recreation experiences, regardless of their migration pathway or status.

For this study, we adopted the definition of recreation endorsed by the National Framework for Recreation in Canada: "Recreation is the experience that results from freely chosen participation in physical, social, intellectual, creative and spiritual pursuits that enhance individual and community well-being" (Canadian Parks and Recreation Association/Interprovincial Sport and Recreation Council [CPRA/ ISRC], 2015, p. 9). This definition acknowledges the characteristics of recreation experiences (enjoyment, freedom, fun, etc.), which are similar qualities ascribed to leisure in academic literature. Throughout this paper, the term leisure will appear if it is used by scholars whose work we are describing, while the term recreation will be used to describe the experiences of participants who engaged in this research, invoking the broad definition of recreation that guided this research.

\section{Literature Review}

Below we summarize key concepts from the literature which have shaped our project, with a particular focus on culture; the role of leisure in transitions, well-being, and belonging; and key barriers and facilitators to recreation participation. This literature review identifies key gaps relating to the need to explore non-Eurocentric (Western) definitions of leisure that are subjectively defined across cultural groups and the exploration of informal and unstructured activities. This project aimed to address these gaps.

\section{The Culturally Bound Nature of Leisure}

The concepts of recreation and leisure have varied meanings cross-culturally. The notion of leisure as 'time' is most often conceptualized in Euro-American contexts as free or unobligated time (Murphy, 1974), with leisure as 'activity' typically referring to activities done in one's free time. It is also common to focus on leisure as experiences characterized by the subjective sense of freedom, involvement, or intrinsic values such as enjoyment or meaning (Mannell \& Kleiber, 1997; Neulinger, 1981). It is debated to what extent these Western conceptualizations reflect 
meanings and definitions of leisure within diverse cultural contexts of perspectives (Kim \& Iwasaki, 2016). Thus, it is important to explore subjective experiences and meanings of leisure to guard against imposition of culturally-bound definitions.

Some leisure and occupational science scholars suggest that the values and meanings associated with different cultures need to be reflected in understandings of daily activities, including leisure (such as the values of individualism versus collectivism; e.g. Hammell, 2014; Walker, 2010) whereas others argue that conceptualizations (and the study) of leisure should take into consideration cultural differences and recognize the complexity and heterogeneity of migrants' experiences, preferences, and cultural practices (Juniu, 2002; Li et al., 2008; Mata-Codesal et al., 2015). Further to this, some researchers challenge the "Western" idea that leisure is an inherently positive experience which can improve life satisfaction, health, and well-being for cultural minorities (Höglhammer et al., 2015), suggesting that narrow, Eurocentric definitions of leisure can exclude and/or serve to limit certain groups. To address this, Hurly and Walker (2019) suggest attending to ethnocultural and individual preferences for leisure, as well as considerations for what is appropriate and meaningful for many ethnocultural groups.

\section{Leisure's Role in Transition}

Resettlement, as experienced by newcomers, constitutes change, transition, and often, disruption in one's routine (Suto, 2013). In framing resettlement as a transition, we focus on changes in daily roles, routines, and environments for newcomers during the resettlement process. It is through these transition processes that newcomers negotiate how to participate in their daily lives in new ways. This negotiation can involve abandoning previously engaged in activities, negotiating different meanings for previous activities, or engaging in new activities altogether (Schisler \& Polatajko, 2002; Suleman \& Whiteford, 2013). Literature points to pleasurable, social activities (e.g., leisure activities) as a supporting factor throughout the transition process (Gallant \& Tirone, 2017; Perez et al., 2010; Suto, 2013).

During the process of transition, engaging in leisure can support newcomers in experiencing or discovering different aspects of a new culture, as well as of their own. In other words, leisure can be a path for newcomers to experience integration of existing cultural characteristics or traits with those of their host society (Kim et al., 2016a, 2016b). Integration is often linked to acculturation which refers to a process of undergoing change of existing cultural traits or values while acquiring those of one's host society (Deng et al., 2005). For example, Horghagen and Josephsson (2010) found that asylum seekers engaging in theatre in Norway was a way of becoming visible within their new environment, and Adrian (2013) found that immigrants and refugee populations in the United States of America (U.S.A.) used music making as a dynamic way of acquiring elements of American culture while maintaining and engaging with cultural practices from their home countries. Indeed, leisure has the potential to positively influence newcomers' transitions during resettlement (Kim et al., 2016a, 2016b) as it provides newcomers with opportunities to interact with community members, develop or strengthen language and 
communication skills, explore and understand new cultural aspects of a host nation, and feel a sense of belonging (Campbell et al., 2016). Therefore, understanding how newcomers experience, negotiate, and participate in leisure activities during their transition into a new country, as well as the barriers and facilitators experienced, is central to understanding what can facilitate resettlement. The following sections briefly review literature about the role of leisure or recreation experiences in the transition process.

\section{Leisure's Role in Newcomers' Health and Well-being During Transition}

Engaging in leisure is not only a means of facilitating the transitions associated with migration but can also help newcomers cope with new stressors or life challenges (Stack \& Iwasaki, 2009). Because of change, transition, or challenges experienced upon resettlement, newcomers may experience acculturative stress - a set of behaviours acquired as a result of or as a response to stress related to resettlement and migration, which often contributes to declines in mental health (Hasmi et al., 2014). However, Hasmi et al.'s (2014) study identified that finding social support and experiencing social integration through leisure helped migrants in South Australia manage acculturative stress. Stodolska et al. (2017) also demonstrated that partaking in nature-based leisure or leisure in natural environments facilitated a sense of self-restoration amongst immigrants in the U.S.A. who were experiencing immigration-related stress. Hurly (2019) also proposed that leisure can have "ameliorative effects" (p. 3) on newcomers' lives, particularly due to its potential to reduce stress related to migration (Höglhammer et al., 2015). Indeed, experiencing personal, social, or cultural connectedness through leisure can help newcomers overcome acculturative stress or immigration-related challenges which can, in turn, promote health and well-being (Kim \& Iwasaki, 2016).

\section{Leisure, Transition, and Belonging}

Phelan and Kinsella (2009) pointed to the importance of engaging in meaningful activities as a way to support identity formation, allowing us to understand that the ways we engage and negotiate spending our time are not only important for wellbeing, but also for our own sense of identity and belonging. For example, McCarty et al. (2020) explored the transition of daily activities (referred to as occupations) in the context of refugees resettling in the U.S.A and found that refugees placed a high level of importance on belonging and social networks — and adapted, terminated, or adopted new activities throughout their resettlement process to support these social aims. Similarly, Kim et al., (2016a, 2016b) found that Korean immigrants often brought in their own ways of being and merged this with their new environment, New Zealand, until they felt valued as members of their new society — pointing to a complex process of negotiation through daily activities. Within the Canadian context, Michalski (2001) found that Iraqi refugees' successful resettlement was linked to ease of access to informal support systems, and Martins and Reid (2007) found that key themes of 'fitting in' and 'coping with a new life' were evident for 
new-immigrant women in Canada. Acevedo and Stodolska's (2019) study shows that engagement in leisure activities through student or campus club involvement facilitated positive integration experiences for Latino university students in the U.S.A. On a similar note, Rich et al. (2015) proposed that engagement in recreational sport or physical activity can foster acculturation and social inclusion for newcomers. Thus, leisure may aid newcomers in retaining their cultural heritage or practices within a dominant culture (Kim \& Iwasaki, 2016). At the same time, leisure can serve as a bridge between newcomers' culture and their host society's culture (Kim et al., 2016a, 2016b), reinforcing leisure's role in newcomers' experiences of acculturation and adaptation during transition.

\section{Newcomers' Experiences of Barriers and Facilitators Related to Leisure}

While leisure can support newcomers' transition, health, and sense of belonging, newcomers may experience unique barriers and facilitators related to leisure. According to Li and Stodolska (2018), "Leisure of [newcomers] cannot be understood in separation from the [barriers or] constraints they face" (p. 14) as migration, transition, and resettlement can pose a multitude of factors that can impede, hinder, or cease newcomers' engagement in leisure (Stodolska, 2000). Competing settlement priorities (Block \& Gibbs, 2017) such as work, lack of free time, learning a new language, and economic constraints are some barriers related to leisure that can be experienced by newcomers (Höglhammer et al., 2015; Hurly, 2019; Lovelock et al., 2011). In addition, distance and transportation difficulties (Hurly, 2019; Lovelock et al., 2011), as well as limited social networks, lack of information or knowledge about leisure and recreation services, and childcare, family, and domestic responsibilities (Block \& Gibbs, 2017; Hurly, 2019) can pose additional barriers to newcomers' engagement in leisure. Personal factors likely related to cultural beliefs and traditions, including gender roles, family attitudes, caring duties, and domestic responsibilities can also influence newcomers' leisure experiences (Block \& Gibbs, 2017; Juniu, 2002; Lovelock et al., 2011; Martins \& Reid, 2007). Moreover, perceived discrimination and social exclusion, fear of the unknown natural environments, and feelings of difference (Hurly \& Walker, 2019; Lovelock et al., 2011; Mock et al., 2013; Taylor \& Toohey, 2001) can hinder newcomers from participating in leisure.

Despite these challenges, past research demonstrates how expanding one's social networks and the possibility of making new friends can help newcomers to frequent places like parks or community gardens for leisure (Stodolska et al., 2017). Stodolska (2000) also identified reliving leisure pastimes, preservation of one's culture, and escaping stress or problems as facilitators for newcomers' continued motivation to engage in leisure. Having access to information (in one's home language; Quirke, 2015) about low- or no-cost opportunities and assistance navigating confusing registration processes have also been identified as key facilitators of participation.

In summary, there is a need to understand newcomers' subjective experiences of recreation/leisure and how diverse forms of recreation can be part of the resettlement 
process. Further to this, there is a need to understand factors that serve as both barriers to, and facilitators of, activity participation and meaningful experiences.

\section{Context and Research Question}

This research project was the first phase of a broader project that aimed to develop an innovative peer leadership initiative to support newcomers in connecting to their community through culture and recreation, which was funded by a culture and innovation fund of the provincial government. The first phase, which is the focus of this paper, aimed to (1) identify and share culturally relevant and community-based recreation experiences through photography and (2) to document the key values and perspectives of newcomers related to recreation in both rural and urban areas. The research question that guided this project was, "What are the meanings and experiences of recreation for newcomers?"

Ethical approval was received from the relevant university. The project took place in two Eastern Canadian communities, one urban and one rural, starting in May 2019 with dissemination activities occurring from 2019 to 2021. For logistical and human resources reasons, each step of the project was carried out first at the urban site, with the rural site following several months later.

\section{Methodology}

This exploratory, qualitative study drew on community-based participatory approaches (Minkler \& Wallerstein, 2011) and used photovoice methods, focus groups, and individual interviews. Community-based participatory research principles guided partnerships with community organizations working with newcomers and the use of visually-based participatory approaches that privileged the perspectives of newcomers regardless of English language ability. The Recreation Access for Newcomers (RAN) Partnership, an existing consortium of representatives from approximately 15 local organizations that work with newcomers in the urban centre, served as the community advisory group to provide guidance throughout the project. The project team came from diverse backgrounds and contexts, including members with personal experiences of immigration.

Drawing on the principles of photovoice approaches (Wang \& Burris, 1997), a brief photographic training session was offered, and participants were invited to take photos of their recreational activities and discuss them at subsequent focus groups to identify the key themes. These themes were synthesized into public photo exhibits in the two settings. 


\section{Participants and Recruitment}

Six newcomer Champions (two rural, four urban), who were identified by the two participating partner community organizations as emerging leaders with conversational English skills, assisted with recruitment along with RAN members. The Champions were from Ethiopia, Syria, India, England, and Iran and included four women and two men. Recruitment posters were used to open conversations with potential participants. Interested participants were directed to the project coordinator who provided additional information and gathered informed consent and demographic information.

Participants were newcomers who had lived in either of the study sites between 4 months and 5 years and who had some experience with recreational activities. English language skills were not required and a total of six interpreters (Somali, Ormo, Arabic, Russian, Nepali, and Spanish) assisted in the recruitment processes and research activities. Although participants as young as 10 years could participate if accompanied by an adult, only one teenaged participant engaged in all aspects of the project. Champions were also invited to participate in the photovoice training, photo taking, and discussions. In total, 51 participants (39 in urban and 12 in rural sites, including six Champions) attended the photovoice training and a total of 40 (28 at the urban site and 12 at the rural site, including four Champions) submitted photos and participated in focus groups.

Photovoice participants $(n=40)$ ranged from 14 to 66 years old and were originally from one of the following 13 countries: Afghanistan, Bhutan, the Democratic Republic of the Congo, Eritrea, Ethiopia, Guatemala, India, Morocco, Nepal, the Philippines, Russia, Somali, and Syria. Sixty-eight percent $(n=27)$ of the participants were female, $45 \%(n=18)$ and had been in their Canadian communities for 3 years or less, and $33 \%(n=13)$ required an interpreter.

At the urban site, most participants $(93 \%, n=26)$ had entered Canada through various resettlement refugee programs and the rest were refugee claimants $(7 \%$, $n=2$ ), meaning they were forced to flee their home country because of fear of persecution (UNHCR, 2021). At the rural site, 92\% $(n=11)$ of the participants were economic class immigrants, meaning they chose to migrate rather than faced forced migration, while $8 \%(n=1)$ had come to Canada under family reunification programs.

\section{Data Collection}

The project involved the following steps: (1) photo training, (2) photo submissions, (3) focus groups, and (4) individual Champion interviews. Table 1 summarizes these key data collection steps, the number of participants, and the type of data collected. 


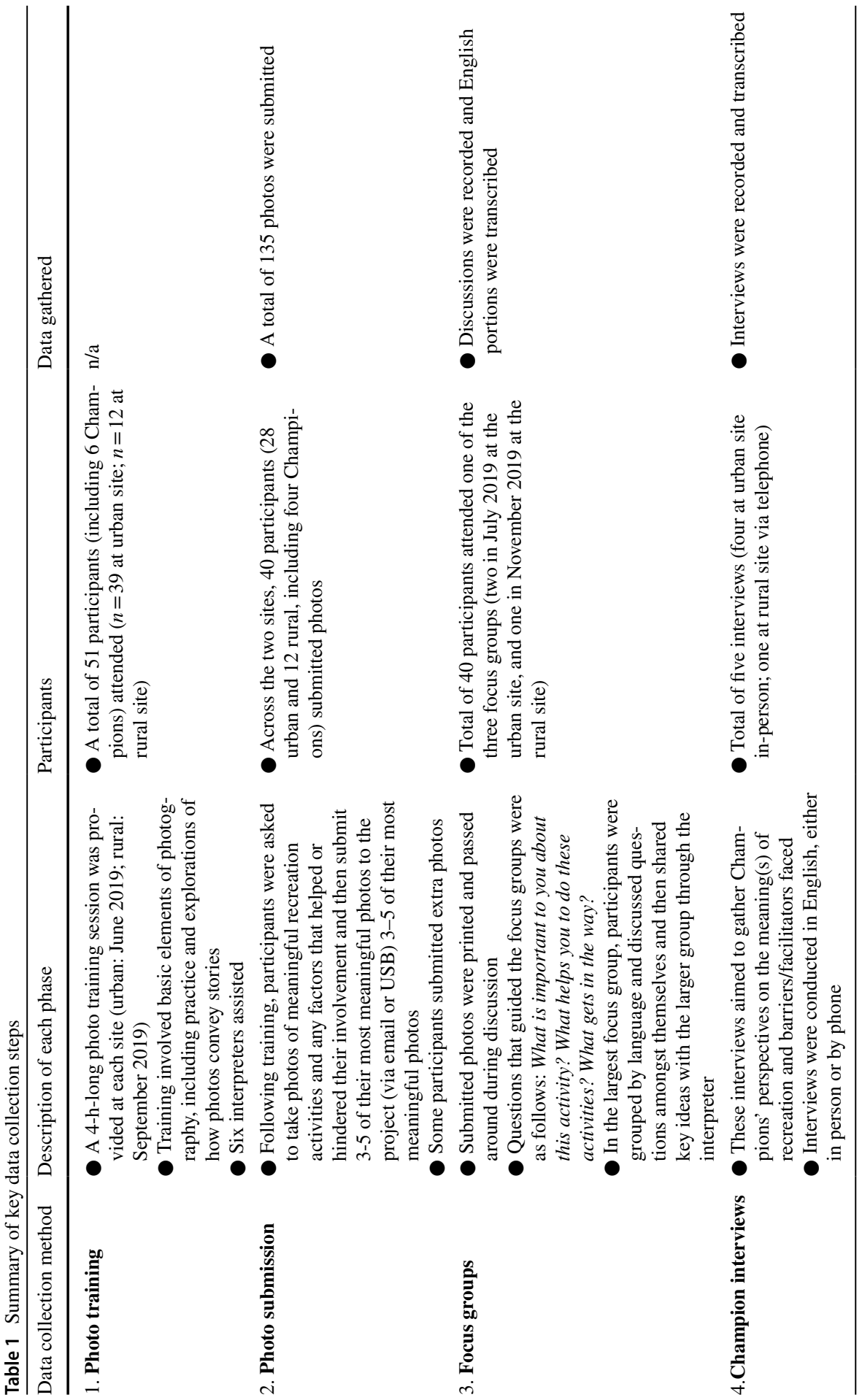




\section{Data Analysis}

Transcripts from the three focus groups and five Champion interviews were analyzed using interpretive description (Thorne, 2016), drawing on constructivist grounded theory analysis methods, using Atlas $T i$ for the cross-case analysis. Following Thorne's suggestion to use analytical scaffolding to begin analysis and then to go beyond that foundation, the analysis was done in two steps: (1) Preliminary descriptive analysis of focus groups by site (urban or rural) that grouped pictures and quotes into general categories for the local photo exhibition and (2) in-depth, cross-case analysis of focus groups and Champion interviews.

At both sites, preliminary descriptive analysis began with one researcher reviewing and coding the transcripts and identifying key themes related to the research questions. At the urban site, these preliminary summaries were shared at an optional group discussion session where participants $(n=13)$ were invited to engage in participatory thematic analysis during which they discussed preliminary themes and confirmed key photos and messages for the photo exhibit. At the rural site, the preliminary data analysis was delayed and the participatory thematic analysis did not occur due to public health restrictions during the COVID-19 pandemic and limited internet access that hindered virtual discussions. The photo exhibition from the urban site was launched in September 2019 at a local library and the rural photo exhibit occurred in March 2021 at a local museum.

Following the preliminary descriptive analysis at each site, focus groups and Champion interview transcripts across both sites were analyzed together by two researchers. The purpose of the cross-case analysis was to deepen our understanding of possible relationships and complexities within and between themes and to identify shared, differing, or overarching themes across sites. Throughout both analysis steps, discussions with the research team served to verify interpretations and alternative interpretations.

\section{Findings}

The participants took pictures of and described a range of recreational activities they engaged in. Although the activities varied, shared themes were distilled into common valued elements and benefits of recreation, which were often intertwined. These valued elements of recreational activities were synthesized into the following key themes: (1) continuity with, and freedom from, past activities and places; (2) being in and connecting with nature; (3) staying physically and mentally well; and (4) connecting and learning with others through reciprocity. These all contributed to the overarching theme that emerged as most meaningful for the participants, (5) developing a sense of belonging: a series of small encounters. Each of these key findings is described below with supporting quotes and examples from participants. We also include a brief summary of (6) barriers and facilitators. Although separated for the sake of clarity and discussion, the themes' interconnectedness is 
discussed. Supporting quotes are provided, indicating if they were from urban or rural participants.

1. Continuity with, and freedom from, past activities and places

Some activities were engaged in because they linked to participants' previous experiences and allowed them to remember their past or continue with an activity from their previous home. For example, activities such as gardening were described as "bring[ing] us our memory" (Urban). Some participants took pictures of gathering mushrooms, biking, or going fishing, which were activities they participated in while living in their previous country. Participants explained they took pictures of landscapes during walks because it was similar to their rural hometowns: "I remember when I do just see something like this [referring to a picture of trees], I remember where I came from... I feel happy and I sense something" (Urban). Photographs of such places or activities from one's previous home allowed people to find "something to connect with our past life" (Rural). In contrast, another participant spoke of a newfound freedom with starting over in Canada where she is "not afraid to do something weird" and thus trying something new is "easier" (Rural) in her new home. She explained: "we make a big start to move from our country to [a] new country and that's why our fears will be left in the past. Now [with this] new life, I can do everything" because "you're free of the ties from your old life" (Rural).

2. Being in and connecting with nature

Many of the recreational activities took place in nature, in the outdoors. Outdoor activities included walking, gardening, fishing, hiking, biking, kayaking, sitting in a park, or playing by the water's edge. Being in nature and feeling connected to nature were important in themselves, because of feelings of "peace," "calm," and "relaxation". Participants spoke of beautiful nearby scenery and places, including beaches, hiking trails, local gardens, and city parks, and the activities they engaged in, such as gathering shells and listening to birds. One participant described enjoying sitting by the water because "water is kind of the source of life, and I'm kind of more refreshed... And then when the ship is on the water, free, just floating, it just makes me ...feel like I'm free and then I can relax in nature" (Urban). Similarly, looking out of a window at a tree while reading a book helped one participant feel "connected to nature...I'm close [to nature] here" (Urban). Key features of many of the outdoor activities that participants talked about were proximity and availability: these activities were close, easy to get to, and free. This was consistent across both the urban and rural sites.

3. Staying physically and mentally well

Building on the sense of relaxation and calm discussed above, participants described recreation being "fun" (Urban), providing a sense of freedom and "giv[ing] meaning to life" (Rural). Recreation was a way to minimize loneliness and thus promote well-being. One participant explained: "sometimes you are far [from home], and you are feeling isolated ... So, all the recreation, it helps in our health.... it helps a lot [with] mental health" (Urban). Participants also spoke of the physical benefits of recreational activities, such as staying healthy through activity. Those who gardened, fished, or gathered mushrooms spoke of 
the health benefits of these foods and activities: "For us [gardening] is very important because we are using a lot of vegetables in our kitchen. And that helps us to stay healthy" (Urban).

4. Connecting and learning with others

The concept of Connecting and learning with others can be seen through various activities photographed by the participants with family and new friends. For example, the participants emphasized the importance of family when describing a photo of gardening together. While they appreciated having healthy food to eat, they stated ".... And the most important thing is that we are doing that as a family" (Urban). A few photos and much discussion surrounded some traditional coffee ceremonies, which brought family and friends together: "[the coffee ceremony] is a way how the neighbours are connected, how the family members are connected... So, all the neighbours come together and drink in one house" (Urban). In addition, the coffee gathering was considered as an event to "provide support and share information" (Urban).

The participants also shared their recreational experiences with new people. Activities such as playing sports, sharing food, or playing in the park allowed them to form connections with people, outside their family, in their new community. Recreational activities were described as the common element that brought diverse people together, as explained when referring to a photo of a cricket match: "[what is] important about cricket is the people who enjoy that sport, they come together and play as a sport and make the network connection" (Rural). The participant explained further, "It's also about the connection .... here all the people, some are from Australia, England and some are from India, they come and play cricket. All are from different cities and different towns. What is common is cricket. It's cricket that makes it get together" (Rural).

Direct interaction or a common language was not necessarily required for the participants to experience connections with others through recreational activities. One participant described through an interpreter joining a group of people already at a gathering: "Even if we are not talking [to] each other, as we observe, we are doing the same thing. That is how we started interacting and communicating..." (Urban).

Some recreational activities also offered the opportunity to learn from others about new cultures or new skills in a safe, non-judgmental context because recreation was considered a "universal language" (Rural). The libraries also provided many opportunities to meet new people to "share our culture, our stories" (Urban). Participants also described some of their recreational activities as a means for English learning and to learn about Canadian culture, such as local festivals or celebrations.

5. Developing a sense of belonging: a series of small encounters

This overarching theme of developing a sense of belonging through a series of small encounters can be seen woven throughout the previous themes. By small encounters, we are referring to activities or experiences that may seem ordinary to others (e.g., sitting on a park bench or meeting for coffee) but were significant or meaningful to participants. Through connections with families and between the past and current self, these small encounters created a sense of feeling welcomed 
and helped to stave off feelings of loneliness, all of which contribute to a sense of belonging. Many participants explained that involvement in recreational activities "gives you that sense of belonging. Like you belong here. This is your new home. And you are welcome as a newcomer" (Urban), and this feeling of belonging can occur "even though we are here, far from home" (Urban). A Champion from the urban site explained, people "belong to something if they are part of that activity. So, I think ...recreation is very important. Very important".

While belonging was mentioned often by the participants, the recreational activities that fostered that sense of belonging were diverse, spanning participation in active team sports to everyday encounters in the community. For example, regular interactions at a local café can create the feeling that "I'm home", and "I'm part of [the] community" (Rural). Small interactions in a park can create a sense of being included in the community:

"[in the park] you will find that inclusion. And then that relationship, the bonding that you may be creating in there. And then the newcomer kid will feel at home. Like "I belong here because I'm playing with another kid" (Urban).

6. Barriers and supports to belonging through recreation

Both personal factors and structural issues were seen to support and limit participation in recreation for these newcomers. Personal facilitators included having the "confidence" and "self-esteem" to be "brave enough to do the first step" to find out more about recreation activities within their communities.

Structural facilitators and barriers included issues of accessibility of activities, spaces, and information; language; familiarity with technology to be able to access online information and registration; other competing demands on time such as shift work; and the availability of social supports, including support from community organizations. Accessibility referred to cost as well as proximity, such as having a park close by or near public transportation. In the rural setting, lack of transportation and amenities was noted. One participant explained, "I like doing things that don't cost money, that's my biggest motivator with recreation because I live very cheap" (Rural). Access to information was interrelated with language. One Champion explained, "The main challenge is not being familiar with the society they live in. They are new here. They don't know what there is." However, language can broker this: "The language barrier of course is huge. Huge....If they have the language, they can read, they can go and talk to people, and, you know, they can be informed" (Urban). Technology was experienced as both a way to "connect us with friends" as well as a barrier when having to navigate complex online registration forms. The availability or lack of free time was also a factor that could limit or facilitate participation in recreation. When reflecting on bringing the coffee ceremony to their local community, one participant noted, "people find it's very busy here because of the work schedule and all that stuff. So, they can't do or prepare coffee as often as they used to. And the second [related challenge] is people are working different shifts. So, it doesn't make it possible to have everyone at the same time [for coffee]" (Urban). And finally, as noted above, having friends or family with whom to do activities was considered a facilitator to engaging in recreation, along with support and information from local community organizations. 


\section{Discussion}

Recreation played diverse roles in the lives of the newcomers who participated in this study, connecting them to the past and to nature, helping them to integrate within their new home, and providing a source of fun, relaxation, socialization, wellness, and overall, belonging. These align with previous scholarship exploring the roles of leisure in the lives of newcomers pertaining to the centrality of social supports and the development of social networks (Li et al., 2015; Suto, 2013), the role of recreation in promoting physical and social well-being (Kim et al., 2017; Kneer et al., 2019), and the importance of nature-based leisure (Hurly \& Walker, 2019). The following discussion focuses on what we believe are the most important learnings generated from this study.

\section{Characteristics of Leisure Valued in Transitions During Resettlement}

The interplay of leisure and everyday activities that emerged in this study helps to illustrate how leisure can contribute to transitions that occur during resettlement. In a scoping review about transitions, Crider and colleagues (2015) identified that participation in meaningful activities, the availability of social supports, and continuity between past and present activities can facilitate transitions. The results of the current study illustrate how the recreational activities identified by participants fulfill many of these functions, and thus appeared to support resettlement processes. Suto (2013) proposed that the social nature of leisure activities described by immigrant women in Canada was central to their development of both social capital (e.g., social networks of reciprocal relationships where information is shared and support provided) and cultural capital (in particular, language development), which are also supported by the findings of the current study. Of note, the qualities of many of these leisure activities described in the current study - low risk, enjoyable, informal, accessible — helped make them doable because they were woven into everyday life. Thus, the leisure activities described in the current study provide examples of how small encounters during leisure have the potential to support transitions associated with resettlement by fostering social networks, supporting physical and mental well-being, and offering safe opportunities for learning. The strong presence of nature-based activities amongst both urban and rural participants in our current study also support the notion that transitions are impacted by environment (Crider et al., 2015), but advance the unique role of accessible nature in supporting well-being and providing a sense of continuity with past places and activities (Hurly \& Walker, 2019; Suto, 2013) that also facilitate transition(s) during resettlement.

While engaging in recreation provided opportunities to feel a sense of continuity to valued memories and activities in participants' home countries, some participants also noted that, having negotiated the challenging process of migration, they felt an openness or freedom to trying new things. Stodolska (2000) 
and Hurly (2019) similarly found that learning new things and engaging in new pastimes were common experiences amongst newcomers. Kleiber et al. (2002) describe the potential of leisure as a means of personal transformation following life events that are characterized by disruption, stress, and adaptation; these are characteristics that could also describe transitions such as immigration (Kim \& Iwasaki, 2016; Stack \& Iwasaki, 2009). These activities often lead to increased receptiveness to, and appreciation of, new experiences, and might explain some participants' openness to novel forms of leisure in our current study.

\section{Leisure's Role in Fostering Belonging}

Sense of belonging seemed to arise from a combination of the features discussed above, such as engaging in activities and places that connect with one's previous home, but also activities and events that allow for meeting new people, learning more about Canada, and exchanging information and experiences. Recreation, because of its fun, often unstructured and casual nature, can provide opportunities that foster these feelings of connection and belonging in a relaxed context. Participation in recreation was at times both casual and meaningful, providing affirmations that one belonged in their new community.

While other researchers have noted the importance of informal social leisure for immigrants (e.g., Hurly, 2019; Juniu, 2002; Stack \& Iwasaki, 2009; Tinsley et al., 2002), participants shared with us that even activities that offered limited opportunities for interaction (e.g., being at a busy festival with family and not knowing others there, nor understanding the language) were nonetheless viewed as meaningful experiences that helped them feel a sense of belonging, and were significant in helping them to acculturate to their new environment, which is supported by similar findings exploring intercultural urban encounters by Ganji and Rishbeth (2020). In other words, feelings of belonging were cultivated through a series of small encounters with family, others, nature, and oneself. It is during these encounters that many participants created and were offered opportunities to integrate their past activities, build social connections and connections to places, learn and exchange experiences, and foster a sense not only of health and wellness, but of belonging within their new home.

The reciprocal nature of recreational activities that involved learning and sharing also draws attention to the potential for bidirectional sharing of cultures and experiences during recreation. A critique of leisure is that it can serve as a way to encourage newcomers to "fit in" by engaging in mainstream activities (Herrero-Arias et al., 2020), thus contributing to acculturation (Kim et al., 2016a, 2016b), a process which historically emphasized changes in newcomers (Nayar, 2015). Bringing an occupational perspective to processes of acculturation in New Zealand, Nayar (2015) emphasized the dynamic, transactional processes of acculturation which occur between the changing daily activities of newcomers and the host country, each influencing and shaping the other. Considering acculturation as a bidirectional, transactional process increases awareness of values, beliefs, and practices that newcomers bring to their new context; recognizes 
that host communities are also constantly changing; and helps both newcomers and host communities acknowledge the variety of cultural influences on interactions. Recognizing acculturation as a transactional process frames resettlement in a new country as an ongoing process and thus can help reduce pressures felt by newcomers to conform as change occurs in both directions (Nayar, 2015). In the current study, this mutual exchange was evident as participants tried recreational activities in their new communities, but also continued with previous activities and emphasized the importance of sharing their own experiences and traditions with others. Some recreational activities appear to allow for such mutual exchange, not only benefitting newcomers, but also influencing and broadening the host community through such exchanges.

\section{Meaningful Small Encounters of Recreation}

Similar to barriers identified in the literature (Höglhammer et al., 2015; Hurly, 2019; Lovelock et al., 2011), some participants in the current study also encountered barriers to recreation, such as cost and transportation, that resulted in some gravitating towards recreation opportunities that could be considered routine and everyday. In other words, participants often described engaging in recreational activities that were close to home and made use of materials and spaces easily accessible to them. Despite the many barriers that limited their options, participants were able to identify diverse, meaningful activities that they perceived as important to their health and well-being during resettlement. This research was novel in highlighting the role of routine, informal leisure activities in the lives of many of these newcomers. Participants valued a range of recreational activities such as gathering with friends or family for coffee, tending a garden, or reading. They described weaving recreation into everyday or obligatory activities, such as by bringing family members to help with gardening and going to the park afterward, using the additional time on a bus transfer to stop at a park, or sitting on a bench in a park listening to birds. This supports the importance of "playfulness", or informal recreation, within public spaces in supporting the integration of recent immigrants (Ganji \& Rishbeth, 2020, p. 231). Further to this, the broad conceptualization of recreation that guided this research could have encouraged participants to consider varied occupations as recreation activities, as compared with Western conceptualizations of recreation that are common in the literature (Hurly, 2019) and focus on structured and organized activities.

\section{Implications for Practice and Research}

This study highlights the diversity of experiences that are considered to be recreation. While recreation practitioners often focus on provision of structured (leaderled) group programs and address well-known barriers impacting newcomers' access to recreation, such as cost and transportation (for example, by accessing subsidized programs), this research suggests that focus should also be on emphasizing nearby, free, and easily available forms of recreation, as well as those where family groups 
can participate together or in parallel activities. In supporting newcomers to infuse recreation into their everyday activities, practitioners could draw on strategies described by participants, including involving family and friends, building in recreation by visiting recreational spaces such as parks in close proximity to appointments or errands, or focusing on relational aspects of activities (i.e., engaging family and friends or noticing a sense of community with those in close proximity). Some participants in this study noted that low-risk opportunities to try informal recreational activities, such as a trial sampler of recreational activities, were appreciated. Such recreation sampler initiatives should be flexible and include opportunities for family members and friends to also participate. Of note, because many valued recreational activities in this study overlapped with or occurred around other activities, this study encourages further reflection on potential ways to include opportunities for informal recreation alongside obligatory activities just as grocery shopping, tending gardens, or doing errands. For example, structured recreation programs as well as service agencies could include information about informal, accessible recreational activities, spaces, and equipment available in a community (such as nearby parks, libraries that offer equipment loans, or indoor play areas), which could assist people in weaving meaningful informal recreational activities into their daily lives. Further interdisciplinary research, in collaboration with service providers and urban planners, for example, could explore how best to structure spaces that could foster such opportunities for leisure as part of daily life. This research suggests that resettlement agencies and practitioners working with newcomers should be attuned to the important role that both recreation and daily, informal activities play in newcomers' lives. Educating immigrant settlement support workers about these benefits may, in turn, help them guide newcomers to prioritize recreation as part of the resettlement transition process. Further to this, it may be beneficial to partner with recreation organizations and/or occupational therapists to deepen opportunities for newcomers to engage in informal, unstructured forms of recreation. In this research, emerging leaders in newcomer communities, referred to in this project as Champions, provided critical support to the project by helping to recruit and support participants throughout the process. Thus, this research highlights the potential of peer support (people with lived experience of immigration) in helping and potentially enhancing the recreation experiences of newcomers.

The methods used in this research also offer insights for those who support newcomers in transition or in accessing recreation. Both photography and storytelling (sharing and describing photos in small groups) were characterized by some participants as valued recreation experiences in and of themselves, and thus offer potential opportunities for many newcomers to build a sense of belonging and connection by focusing attention on everyday enjoyable activities and providing the opportunity to learn and share across cultures.

While recreation and leisure activities were characterized in this research as inherently beneficial or positive, it is critical to note that leisure has also, both historically and in a contemporary context, been used as a means of reinforcing norms of the dominant group. Historically, for example, leisure was organized and regulated to maintain public order, to enforce moral norms, and to ensure the productivity of the workforce (Cassar, 2017; Kelly \& Freysinger, 2000). Currently, organized 
leisure continues to be a means to build a sense of social cohesion and common identity by channelling people, particularly newcomers, into organized leisure activities that reflect mainstream culture (e.g., hockey in Canada), with newcomers' acceptance of these activities sometimes considered a measure of their ability to adapt to, or assimilate to their new homes (Barrick et al., 2021; Spracklen et al., 2015). Consequently, immigrants' participation in unstructured, self-organized leisure activities can be perceived by some as problematic or suspicious (Agergaard et al., 2016), rather than valued as acts of agency and ways to contribute to their new communities. Leisure choice can thus be a source of tension as immigrants balance their pursuit of meaningful leisure with expectations to "fit in" by engaging in mainstream leisure activities (Herrero-Arias et al., 2020). While the current study did not aim to examine this tension, by centering the subjective definitions of newcomers, it begins to offer alternatives to mainstream leisure activities which can foster further critical self-reflection of biases and assumptions of what leisure can or should involve.

Finally, this research highlights the need for attention to recreation that occurs outside organized recreation programs, such as amongst families and individually. For example, there was some indication in our research that the cumulative impact of small encounters was powerful, but this area requires further study. Extending from this, there is a need for future research to understand the range of strategies newcomers use to weave leisure into their everyday lives, and to build on these lived experiences to develop strategies that would make leisure more accessible to other newcomers, particularly those who might face barriers to recreation (e.g., due to transportation, shift work, disability). Of particular interest is the observation that for some participants, brief encounters, even when there was not a shared language, seemed to support feelings of belonging. Exploration of the weaving of recreational activities with other daily activities could help further expand understandings of leisure across cultures to get at the qualities and features that are subjectively experienced during activities. While there were some possible emerging differences across the urban and rural sites (such as differences in access to public transportation, differences in immigration status and thus employment), this project did not seek to uncover competing perspectives between groups, but rather to explore common understandings of leisure based on the subjective experiences of newcomers. The broad definition of recreation used in this project made it possible for all participants to identify meaningful leisure experiences within their everyday lives. Although beyond the scope of this project, there is merit in future research that examines whether there are gender differences in their meanings, differences between those who came to Canada through different mechanisms (e.g. refugee versus economic migrants), or differences in settlement in urban and rural locations.

\section{Limitations}

Although the participatory processes used in this research are a strength of this work, because of public health restrictions associated with the COVID-19 pandemic, we were not able to meet with participants at the rural site to engage 
them in participatory analysis of themes associated with their photos. A second limitation is that the research leads are not newcomers and thus approached this research from the perspective of the host culture. However, our research team is a diverse team including members with immigration experiences who shared their perspectives and experience throughout the research process. In addition, we regularly consulted with a project advisory team for advice and guidance. The third limitation pertains to recruitment strategies which occurred through partner organizations, which may not have reached those not already connected to such services. The fourth limitation relates to the aim of photovoice methodology to engage decision makers with key messages. While recreation and immigration services staff and government officials attended the urban photo exhibit launch in 2019, because of public health restrictions, key decision makers were not actively engaged during the rural launch in 2021. Fifth, asking people to take pictures and share them with others as part of this research created opportunities for participants to notice recreation as they experienced it in their everyday lives, and may have guided participants to identify informal, everyday forms of recreation and opportunities for sharing as meaningful. Lastly, while participants were diverse in age, gender, and time in country, this project did not focus on potential differences across these factors, but instead aimed to explore commonalities across diverse leisure experiences. As a result of this focus, potential differences in access to some recreation activities were not explored.

\section{Conclusion}

This research engaged newcomers, through photo taking and photo sharing, in identifying recreation experiences that were meaningful to them. The data described the importance of recreation in terms of providing a sense of continuity with, and freedom from, past activities and places, connecting with nature, staying physically and mentally well, and learning with others through reciprocity. These all contributed to the overarching theme that emerged as most meaningful for the participants, developing a sense of belonging: a series of small encounters. The findings highlight the powerful role of recreation within the resettlement process, and highlight particularly the role of small, informal recreational experiences that are woven into everyday lives and routines. Such experiences contribute to a sense of belonging for newcomers, thus assisting the resettlement process.

Acknowledgements We thank our community partners for their ongoing contributions to this project: Recreation Access for Newcomers, Immigrant Services Association of Nova Scotia, Halifax Public Libraries, South Shore Multicultural Association, Halifax Immigration Partnership, and YMCA and Halifax Parks and Recreation. We also thank the newcomer Champions and participants of this project for taking their time to share their experiences with us.

Author Contribution Gallant, Hutchinson, and Lauckner contributed to the conceptualization of the project, grant submission, ethics submission, data collection, data analysis, and manuscript preparation, with 
particular focus on "Literature Review" (Hutchinson, Gallant), "Methodology" (Lauckner), Findings (Lauckner), and "Discussion" (Gallant, Hutchinson, Lauckner). Akbari served as the project coordinator, led recruitment, and assisted with data collection, analysis and manuscript preparation, with particular focus on "Methodology". Tomas assisted with literature review and manuscript preparation, with particular focus on the "Literature Review" section. Pride contributed to data analysis and manuscript preparation, in particular the "Findings" section. All authors have contributed substantially to the writing of the article.

Funding Funding for this project was received from the Province of Nova Scotia, Department of Communities, Culture and Heritage (Support4Culture Program) and Dalhousie University's Faculty of Health and School of Occupational Therapy.

\section{Code Availability n/a.}

\section{Declarations}

Ethics Approval Approval received from Dalhousie University Research Ethics Board (\# 2019 4709).

Consent to Participate Informed consent was received by all participants prior to their participation in the project.

Consent for Publication Informed consent included a statement regarding publication and all authors consent to and have participated in this publication.

Conflict of Interest The authors declare no competing interests.

\section{References}

Acevedo, J. C, Stodolska, M. (2019). Empowerment, resistance, and leisure among Latino student organizations. Leisure Sciences 41(6), 460-476 https://doi.org/10.1080/01490400.2017.1378141

Adrian, A. (2013). An exploration of Lutheran musicmaking among US immigrant and refugee populations. Journal of Occupational Science, 20(2), 160-172. https://doi.org/10.1080/14427591. 2013.775690

Agergaard, S., la Cour, A. M., \& Gregersen, M. T. (2016). Politicisation of migrant leisure: A public and civil intervention involving organized sports. Leisure Studies, 35(2), 200-214. https://doi.org/ 10.1080/02614367.2015.1009848

Barrick, S., Bridel, W., Miller, J. B. (2021). Striving for newcomer inclusion: A critical analysis of Canadian Intro to Sport programmes. Leisure/Loisir. Advance online publication. https://doi.org/10. 1080/14927713.2021.1872406

Block, K., Gibbs, L. (2017). Promoting social inclusion through sport for refugee-background youth in Australia: Analysing different participation models. Social Inclusion, 5(2), 91-100. https://doi.org/ 10.17645/si.v5i2.903

Caidi, N., \& Allard, D. (2005). Social inclusion of newcomers to Canada: An information problem? Library \& Information Science Research, 27(3), 302-324. https://doi.org/10.1016/j.lisr.2005.04.003

Caldwell, L. L. (2005). Leisure and health: Why is leisure therapeutic? British Journal of Guidance and Counselling, 33(1), 7-26. https://doi.org/10.1080/03069880412331335939

Campbell, G., Glover, T., \& Laryea, E. (2016). Recreation, settlement, and the welcoming community: Mapping community with African-Canadian youth newcomers. Leisure Sciences, 38(3), 215-231. https://doi.org/10.1080/01490400.2015.1087896

Canadian Parks and Recreation Association and Interprovincial Sport and Recreation Council. (2015). A framework for recreation in Canada: Pathways to wellbeing. Ottawa: Canadian Recreation and Parks Association. https://static1.squarespace.com/static/57a2167acd0f68183878e305/t/5926efaceb bd1a74b7b584d8/1495723950196/Framework+For+Recreation+In+Canada_2016+w+citation.pdf 
Cassar R (2017) Leisure and hegemony. In K. Spracklen, E. Sharpe, B. Lashua, \& S. Swain (Eds.), The Palgrave handbook of leisure theory (pp. 539-556). Palgrave Macmillan. https://doi.org/10.1057/ 978-1-137-56479-5_31

Crider, C., Calder, C., Bunting, K., \& Forwell, S. (2015). An integrative review of occupational science and theoretical literature exploring transition. Journal of Occupational Science, 22(3), 304-319. https://doi.org/10.1080/14427591.2014.922913

Deng, J., Walker, G., \& Swinnerton, G. (2005). Leisure attitudes: A comparison between Chinese in Canada and Anglo-Canadians. Leisure/loisir, 29(2), 239-273. https://doi.org/10.1080/14927713.2005. 9651331

Ganji, F., \& Rishbeth, C. (2020). Conviviality by design: The socio-spatial qualities of spaces of intercultural urban encounters. Urban Design International, 25, 215-234. https://doi.org/10.1057/ s41289-020-00128-4

Gallant, K., \& Tirone, S. (2017). A 'good life without bells and whistles': A case study of immigrants' well-being and leisure and its role in social sustainability in Truro, Nova Scotia. Leisure/loisir, 41(3), 423-442. https://doi.org/10.1080/14927713.2017.1352456

Ghahari, S., Lui, J., Nagra, S., \& Morassaei, S. (2019). The life experiences of refugees in Canada: A comprehensive scoping review to identify unmet needs and barriers. Journal of International Migration and Integration, 21, 1249-1261. https://doi.org/10.1007/s12134-019-00727-3

Hammell, K. R. (2014). Belonging, occupation, and human well-being: An exploration. Canadian Journal of Occupational Therapy, 81(1), 39-50. https://doi.org/10.1177/0008417413520489

Hasmi, H. M., Gross, M. J., \& Scott-Young, C. M. (2014). Leisure and settlement distress: The case of South Australian migrants. Annals of Leisure Research, 17(4), 377-397. https://doi.org/10.1080/ 11745398.2014.948023

Herrero-Arias, R., Lee, E., \& Hollekim, R. (2020). 'The more you go to the mountains, the better parent you are'. Migrant parents in Norway navigating risk discourses in professional advice on family leisure and outdoor play. Health, Risk, \& Society, 22(7-8), 403-420. https://doi.org/10.1080/13698 575.2020 .1856348

Höglhammer, A., Stokowski, P., Muhar, A., Schauppenlehner, T., Yalcintepe, E., \& Renner, J. (2015). Experiences and meanings of leisure for members of the Turkish and Chinese communities in Vienna, Austria. World Leisure Journal, 57(3), 196-208. https://doi.org/10.1080/16078055.2015. 1066602

Horghagen, S., \& Josephsson, S. (2010). Theatre as liberation, collaboration and relationship for asylum seekers. Journal of Occupational Science, 17(3), 168-176. https://doi.org/10.1080/14427591.2010. 9686691

Hurly, J. (2019). 'I feel something is still missing': Leisure meanings of African refugee women in Canada. Leisure Studies, 38(1), 1-14. https://doi.org/10.1080/02614367.2018.1515241

Hurly, J., \& Walker, G. (2019). "When you see nature, nature give you something inside": The role of nature-based leisure in fostering refugee well-being in Canada. Leisure Sciences, 41(4), 260-277. https://doi.org/10.1080/01490400.2017.1325799

Juniu, S. (2002). Perception of leisure in Latino women immigrants. World Leisure Journal, 44(1), 48-55. 04419057.2002.9674260

Kelly, J. R., Freysinger, V. J. (2000). $21^{\text {st }}$ century leisure: Current issues. Pearson.

Kim, J., \& Iwasaki, Y. (2016). Role of leisure-generated meanings in adaptation to acculturation stress of Korean immigrants in Canada. Loisir Et Société / Society and Leisure, 39(2), 177-194. https://doi. org/10.1080/07053436.2016.1198592

Kim, J., Heo, J., Dvorak, R., Ryu, J., \& Han, A. (2017). Benefits of leisure activities for health and life satisfaction among Western migrants. Annals of Leisure Research, 21(1), 47-57. https://doi.org/10. $1080 / 11745398.2017 .1379421$

Kim, J., Heo, J., \& Lee, C. (2016a). Exploring the relationship between types of leisure activities and acculturation among Korean immigrants. Leisure Studies, 35(1), 113-127. https://doi.org/10.1080/ 02614367.2015.1055295

Kim, H., Hocking, C., McKenzie-Green, B., \& Nayar, S. (2016b). Occupational experiences of Korean immigrants settling in New Zealand. Journal of Occupational Science, 23(2), 181-195. https://doi. org/10.1080/14427591.2015.1126168

Kleiber, D. A., Hutchinson, S. L., \& Williams, R. (2002). Leisure as a resource in transcending negative life events: Self-protection, self-restoration, and personal transformation. Leisure Sciences, 24(2), 219-235. https://doi.org/10.1080/01490400252900167 
Kneer, J., van Eldik, A. K., Jansz, J., Eischeid, S., Usta, M. (2019). With a little help from my friends: Peer coaching for refugee adolescents and the role of social media. Media and Communication, 7(2), 264-274. https://doi.org/10.17645/mac.v7i2.1876

Li, C., Absher, J. D., Graefe, A. R., \& Hsu, Y. (2008). Services for culturally diverse customers in parks and recreation. Leisure Sciences, 30(1), 87-92. https://doi.org/10.1080/01490400701756493

Li, K., Sotiriadou, P., \& Auld, C. (2015). An examination of the role of sport and leisure on the acculturation of Chinese immigrants. World Leisure Journal, 57(3), 209-220. https://doi.org/10.1080/16078 055.2015 .1066603

Li, M. Z., Stodolska, M. (2018). Leisure as a constraint and a manifesto for empowerment: The life story of a Chinese female migrant worker. Leisure Sciences. [Advance online publication] https://doi.org/ 10.1080/01490400.2018.1483854

Lovelock, K., Lovelock, B., Jellum, C., \& Thompson, A. (2011). In search of belonging: Immigrant experiences of outdoor nature-based settings in New Zealand. Leisure Studies, 30(4), 513-529. https:// doi.org/10.1080/02614367.2011.623241

Mata-Codesal, D., Peperkamp, E., \& Tiesler, N. (2015). Migration, migrants and leisure: Meaningful leisure? Leisure Studies, 34(1), 1-4. https://doi.org/10.1080/02614367.2015.992620

Martins, V., \& Reid, D. (2007). New-immigrant women in urban Canada: Insights into occupation and sociocultural context. Occupational Therapy International, 14(4), 203-220. https://doi.org/10.1002/ oti. 233

Mannell, R. C., Kleiber, D. A. (1997) A social psychology of leisure. State College, PA: Venture.

McCarty, K., Cantrell, J.-E., Daine, J., Banuelos, K. K., \& Chan, A. (2020). Transition in occupations of refugees during resettlement. Open Journal of Occupational Therapy, 8(4), 1-15. https://doi.org/10. $15453 / 2168-6408.1714$

Michalski, J. H. (2001). The challenges of resettlement among male, government-assisted Iraqi refugees in Canada. Journal of Social Work Research and Evaluation, 2(2), 207- 226. https://psycnet.apa. org/record/2002-02526-007

Minkler, M., Wallerstein, N (Eds.). (2011). Community-based participatory research for health: From process to outcomes. 2nd Ed. John Wiley \& Sons.

Mock, S. E., Wilson, A. W., Smale, B., \& Hilbrecht, M. (2013). The association of physically active leisure with well-being among diverse racial groups. Leisure/loisir, 37(3), 287-301. https://doi.org/10. 1080/14927713.2013.867159

Murphy, J. F. (1974). Concepts of leisure: Philosophical implications. Prentice Hall.

Nayar, S. (2015). Resituating models of acculturation: An occupational dimension. Journal of International Migration and Integration, 16(4), 1141-1155. https://doi.org/10.1007/s12134-014-0379-8

Neulinger, J. (1981) The psychology of leisure Thomas.

Perez, A., Keller, C., \& Nunez, M. (2010). Leisure, health, and adjustment of immigrants. In L. Payne, B. Ainsworth, \& G. Godbey (Eds.), Leisure, health, and wellness: Making the connections (pp. 373381). Venture.

Phelan, S., \& Kinsella, E. A. (2009). Occupational identity: Engaging socio-cultural perspectives. Journal of Occupational Science, 16(2), 85-91. https://doi.org/10.1080/14427591.2009.9686647

Quirke, L. (2015). Searching for joy: The importance of leisure in newcomer settlement. Journal of International Migration and Integration, 16(2), 237-248. https://doi.org/10.1007/s12134-014-0388-7

Rich, K. A., Misener, L., \& Dubeau, D. (2015). "Community cup, we are a big family": Examining social inclusion and acculturation of newcomers to Canada through a participatory sport event. Social Inclusion, 3(3), 129-141. https://doi.org/10.17645/si.v3i3.141

Schisler, A. C., \& Polatajko, H. (2002). The individual as mediator of the person-occupation-environment interaction: Learning from the experience of refugees. Journal of Occupational Science, 9(2), 82-92. https://doi.org/10.1080/14427591.2002.9686496

Spracklen, K., Long, J., \& Hylton, K. (2015). Leisure opportunities and new migrant communities: Challenging the contributions of sport. Leisure Studies, 34(1), 114-129. https://doi.org/10.1080/02614 367.2014 .939989

Stack, A. C., \& Iwasaki, Y. (2009). The role of leisure pursuits in adaptation processes among Afghan refugees who have immigrated to Winnipeg, Canada. Leisure Studies, 28(3), 239-259. https://doi. org/10.1080/02614360902951658

Statistics Canada (2010). Canada's Ethnocultural Mosaic, 2006 Consensus: Definitions. Statistics Canada. https://www.mjseatery.com/menu.php

Stodolska, M. (2000). Changes in leisure participation patterns after immigration. Leisure Sciences, 22, 39-62. https://doi.org/10.1080/014904000272966 
Stodolska, M., Peters, K., \& Horolets, A. (2017). Immigrants' adaptation and interracial/interethnic interactions in natural environments. Leisure Sciences, 39(6), 475-491. https://doi.org/10.1080/01490 400.2016.1213676

Suleman, A., \& Whiteford, G. E. (2013). Understanding occupational transitions in forced migration: The importance of life skills in early refugee resettlement. Journal of Occupational Science, 20(2), 201-210. https://doi.org/10.1080/14427591.2012.755908

Suto, M. (2013). Leisure participation and well-being of immigrant women in Canada. Journal of Occupational Science, 20(1), 48-61. https://doi.org/10.1080/14427591.2012.732914

Taylor, T., \& Toohey, K. (2001). Behind the veil: Exploring the recreation needs of Muslim women. Leisure/loisir, 26(1), 85-105. https://doi.org/10.1080/14927713.2001.9649930

Tinsley, H. E., Tinsley, D. J., \& Croskeys, C. E. (2002). Park usage, social milieu, and psychosocial benefits of park use reported by older urban park users from four ethnic groups. Leisure Sciences, 24(2), 199-218. https://doi.org/10.1080/01490400252900158

Thorne, S. (2016). Interpretive description: Qualitative research for applied practice. Routledge.

UNHCR. (2021). What is a refugee. UNHCR: The UN Refugee Agency website. Retrieved May 15, 2021 from https://www.unhcr.org/what-is-a-refugee.html

Walker, G. J. (2010). The effects of personal, contextual, and situational factors on the facilitation of intrinsic motivation: The case of Chinese/Canadians. Journal of Leisure Research, 42(1), 43-66. https://doi.org/10.1080/00222216.2010.11950194

Wang, C., \& Burris, M. A. (1997). Photovoice: Concept, methodology, and use for participatory needs assessment. Health Education \& Behavior, 24(3), 369-387. https://doi.org/10.1177/1090198197 02400309

Publisher's Note Springer Nature remains neutral with regard to jurisdictional claims in published maps and institutional affiliations. 